\title{
Clinicopathological Difference and Survival Impact of Patients with c-SCLC and SCLC
}

\begin{abstract}
Chenyue Zhang, ${ }^{1,2, *}$ Xiaoling Shang, ${ }^{3,4, *}$ Jian Sun, ${ }^{5}$ Zhenxiang Li, (iD ${ }^{6}$ Jiamao Lin, ${ }^{7}$ Chenglong Zhao, ${ }^{8}$

Haiyong Wang $\mathbb{D}^{7}$

'Department of Integrated Therapy, Fudan University Shanghai Cancer Center, Shanghai, 200032, People's Republic of China;

${ }^{2}$ Department of Oncology, Shanghai Medical College, Fudan University, Shanghai, People's Republic of China; ${ }^{3}$ Department of Clinical Laboratory, Shandong Cancer Hospital and Institute, Shandong First Medical University and Shandong Academy of Medical Sciences, Jinan, 250I I7, People's Republic of China;

${ }^{4}$ Department of Clinical Laboratory, Shandong University, Jinan, 2500 I2, People's Republic of China; ${ }^{5}$ Department of Thoracic Surgery, Shandong Cancer Hospital and Institute, Shandong First Medical University and Shandong Academy of Medical Sciences, Jinan, 250I I7, People's Republic of China;

${ }^{6}$ Department of Radiation Oncology, Shandong Cancer Hospital and Institute, Shandong First Medical University and Shandong Academy of Medical Sciences, Jinan, 250I I7, People's Republic of China; ${ }^{7}$ Department of Internal Medicine-Oncology, Shandong Cancer Hospital and Institute, Shandong First Medical University and Shandong Academy of Medical Sciences, Jinan, 250I I7, People's Republic of China; ${ }^{8}$ Department of Pathology, Shandong Cancer Hospital and Institute, Shandong First Medical University and Shandong Academy of Medical Sciences, Jinan, 250 I I7, People's Republic of China

*These authors contributed equally to this work
\end{abstract}

Correspondence: Chenyue Zhang Department of Integrated Therapy, Fudan University Shanghai Cancer Center, Shanghai, 200032, People's Republic of China Email zhangchenyue_yazi@I26.com

Haiyong Wang

Department of Internal Medicine-Oncology, Shandong Cancer Hospital and Institute, Shandong First Medical University and Shandong Academy of Medical Sciences, Jinan, 250 I I7, People's Republic of China Tel/Fax +86 531 67626332

Email wanghaiyong6688@I26.com
Background: Combined small cell lung cancer (c-SCLC) distinguishes itself from small cell lung cancer (SCLC) due to its inclusion of both SCLC and non-small cell lung cancer (NSCLC) components. Few studies have compared clinicopathological characteristics, prognosis and factors affecting survival. We therefore addressed the issues in this study.

Patients and Methods: A total of 400 c-SCLC and 20,841 SCLC patients were enrolled using SEER database. Difference in clinicopathological characteristics of SCLC and c-SCLC patients was analyzed using chi-square. Kaplan-Meier was applied to compare their survival before and after propensity score matching (PSM). Cox regression model was adopted to assess the impact of different clinical variables on survival. Logistic regression was applied to identify risk factors for c-SCLC and SCLC patients.

Results: Differences in race, sex, T stage, N stage, surgery, bone, brain and liver metastasis were detected between c-SCLC and SCLC patients. c-SCLC patients had better overall survival (OS) than SCLC patients before PSM. Age, race, sex, T stage, N stage, surgery, bone, brain, liver and lung metastasis were prognostic factors affecting OS for c-SCLC and SCLC $(\mathrm{P}<0.05)$. However, a significant OS benefit was not observed in c-SCLC after adjusting for clinicopathological variables (HR, 0.950; 95\% CI, 0.842-1.073; $P=0.411$ ). No significant OS difference was found between c-SCLC and SCLC patients after PSM ( $\mathrm{P}=0.789)$. c-SCLC patients had lower risk of lymph node (OR: 0.555; 95\% CI: 0.439-0.703; P < 0.001) and liver metastasis (OR: 0.591; 95\% CI: $0.448-0.779$; $\mathrm{P}<0.001)$, whereas had no significant differences in bone and brain metastasis risks $(\mathrm{P}>0.05)$ compared with SCLC patients.

Conclusion: The prognosis of c-SCLC did not significantly differ from that of SCLC if clinicopathological characteristics are controlled. Better prognosis for c-SCLC patients over SCLC patients may be ascribed to fewer liver and lymph node metastases upon diagnosis.

Keywords: prognosis, overall survival, clinicopathological features, c-SCLC, SCLC

\section{Background}

Small cell lung cancer (SCLC) accounts for $15-20 \%$ in all types of lung cancer. ${ }^{1-3}$ And the World Health Organization (WHO)/International Association for the Study of Lung Cancer (IASLC) classification has divided SCLC into two subtypes: pure SCLC and c-SCLC. ${ }^{4}$ c-SCLC was defined as SCLC combined with any of nonsmall cell lung cancer (NSCLC) histological types. And NSCLC can be squamous cell carcinoma, adenocarcinoma, large cell neuroendocrine carcinoma and so forth. The components of c-SCLC could be the combination of SCLC with several histological types of NSCLC, with the SCLC combined with squamous cell carcinoma as the most common type. The incidence of c-SCLC was much lower than that of pure SCLC, which could be attributed to insufficient diagnostic information. One important factor is that samples acquired from small biopsy and cytological 
analyses are rather limited. Previous studies have shown that c-SCLC may have distinct biological behavior compared with pure SCLC. ${ }^{5,6}$ However, most of these studies either refer to SCLC as pure SCLC, or confuse SCLC with c-SCLC. ${ }^{7,8}$

Considering the shortfalls of previous studies, we screened 400 patients diagnosed with c-SCLC and 20,841 patients diagnosed with SCLC from 2010 to 2015 using SEER database. We aimed to compare the clinicopathological difference and survival impact of patients with c-SCLC and SCLC.

\section{Patients and Methods}

\section{Data Source}

We used the SEER*Stat software to identify the appropriate patients. ${ }^{9}$ In the present study, c-SCLC was defined as SCLC combined with any of NSCLC histological types, such as squamous cell carcinoma, adenocarcinoma, large cell neuroendocrine carcinoma and so forth. In c-SCLC, the combined components may contain one or more of NSCLC histological types. According to the Union for International Cancer Control (UICC) and the American Joint Committee on Cancer (AJCC) seventh edition TNM stage, we screened 400 patients diagnosed with c-SCLC and 20,841 patients diagnosed with SCLC from 2010 to 2015 . The included patients should meet the following criteria: the C-SCLC and SCLC diagnosis was pathologically confirmed, with active follow-up, with only one primary tumor and with or without surgical treatment. Patients with benign or borderline tumors, unknown TNM stage and the ambiguous information of clinicopathological features and survival were all excluded. Our study was mainly based on the SEER database. Institutional approval and patient consents were not needed due to open access to the public database, according to the stipulations by Shandong Cancer Hospital and Institute, Fudan University Shanghai Cancer Center.

\section{Statistical Analysis}

For all patients with c-SCLC and SCLC, the following variables were analyzed including Age, Race, Sex, T stage, $\mathrm{N}$ stage, Surgery, Bone metastasis, Brain metastasis, Liver metastasis and Lung metastasis. In this study, OS was regarded as the primary endpoint. The chi-square test was used to analyze the differences in baseline characteristics among the included patients.
In addition, propensity score matching (PSM) was used to eliminate any bias between patients with c-SCLC and SCLC. Using the chi-square test, the data on Age, Race, Sex, T stage, N stage, Bone metastasis, Brain metastasis, Liver metastasis and Lung metastasis were included in the propensity model to generate a matching ratio of 1:1.

The Kaplan-Meier and Log rank tests were used to analyze the survival curves. The univariate and multivariate Cox proportional hazards regression models were used to evaluate different variables affecting OS. Risk factor analysis of metastasis pattern for patients with c-SCLC compared with SCLC was conducted using multivariate logistic regression. A P $<0.05$ was considered statistically significant. The statistical software SPSS 22.0 (SPSS, IL, Chicago) was used for data analysis.

\section{Results}

\section{Clinicopathological Characteristics of Patients with SCLC and c-SCLC}

We first analyzed the difference in clinicopathological characteristics between c-SCLC and SCLC patients. A total of 20,841 SCLC patients and 400 c-SCLC patients from the SEER database from 2010 to 2015 were included for analysis. Of all these patients, the proportion of was $1.9 \%$ for c-SCLC and $98.1 \%$ for SCLC. Compared with SCLC patients, there were more patients with $\mathrm{T} 1$ disease $(21.5 \%$ vs $12.1 \%)$ and fewer with $\mathrm{T} 4$ stage $(27.3 \%$ vs $38.6 \%$ ) among c-SCLC patients. Similarly, the proportion of patients with N0-N1 stage $(42.5 \%)$ was higher in c-SCLC patients than that in SCLC patients $(21.8 \%)$. However, in contrast, the proportion of N2-N3 stages patients was lower in c-SCLC (57.5\%) than that in SCLC (78.1\%). In addition, for c-SCLC patients, there were fewer bone metastasis $(15.5 \%$ vs $23.3 \%)$, brain metastasis (11.8\% vs $16.7 \%)$ and liver metastasis $(16.0 \%$ vs $30.2 \%)$ compared with SCLC patients. And patients with c-SCLC (26.5\%) were more likely to undergo surgery than patients with SCLC (2.4\%). Our result showed that there was no significant difference in age $(\mathrm{P}=0.158)$ and lung metastasis $(P=0.291)$ between SCLC and c-SCLC patients. However, there were significant differences in race $(\mathrm{P}=0.030)$, sex $(\mathrm{P}=0.018)$, $\mathrm{T}$ stage $(\mathrm{P}<0.001)$, $\mathrm{N}$ stage $(\mathrm{P}<0.001)$, surgery $(\mathrm{P}<0.001)$, bone metastasis $(\mathrm{P}<0.001)$, brain metastasis $(\mathrm{P}=0.008)$ and liver metastasis $(\mathrm{P}<0.001)$ between them. The baseline characteristics of these patients are shown in Table 1 . 


\section{Survival Analysis for Patients with c-SCLC and SCLC Before PSM}

Next, the survival was compared between patients with c-SCLC and SCLC. Kaplan-Meier analysis and Log rank test were used to compare survival differences between c-SCLC and SCLC patients. Then, the respective survival curves of these patients were depicted before PSM. The result revealed that c-SCLC patients' survival was significantly longer than that of SCLC patients $(\mathrm{P}<0.001)$ (Figure 1). Multivariate Cox regression analyses were further applied to determine prognostic factors for OS in patients with c-SCLC and SCLC. Results have shown that age, race, sex, T stage, $\mathrm{N}$ stage, surgery, bone metastasis, brain metastasis, liver metastasis and lung metastasis are important factors that would affect OS for patients with SCLC and c-SCLC (P < 0.05). However, cancer type was not an independent prognostic factor for OS for patients with SCLC and c-SCLC (hazard ratio (HR), 0.950; 95\% confidence interval (CI), 0.842-1.073; $\mathrm{P}=0.411$ ). These results indicated that patients with c-SCLC did not gain an obvious survival advantage compared with SCLC after adjusting for clinicopathological variables including age, race, sex, $\mathrm{T}$ stage, $\mathrm{N}$ stage, surgery, bone metastasis, brain metastasis, liver metastasis and lung metastasis (Table 2).

\section{Survival Analysis for Patients with c-SCLC and SCLC After PSM}

Before PSM, there were 400 c-SCLC patients and 20,841 SCLC patients included in the present study. In order to better analyze the influence of clinical variables on OS, we conducted PSM to eliminate bias between patients with c-SCLC and SCLC. After 1:1 matching, a cohort of 400 patients were enrolled in each group. In addition to surgery, the ratio of patients with other clinical characteristics was divided on average between c-SCLC and SCLC patients, as demonstrated in Table 3.

Then, we further depicted the survival curves for c-SCLC and SCLC patients after PSM. Results showed that there was no significant difference in OS between c-SCLC patients and SCLC patients $(\mathrm{P}=0.789)$ (Figure 2A). Similarly, the survival curves for patients with c-SCLC and SCLC undergoing surgical treatments were depicted. It was also shown that there was no significant difference in OS between patients with c-SCLC and SCLC after surgery $(\mathrm{P}=0.162)$ (Figure $2 \mathrm{~B})$.
Table I Combined Small Cell Lung Cancer and Small Cell Lung Cancer Patient Characteristics from SEER Database

\begin{tabular}{|c|c|c|c|}
\hline Variables & SCLC (\%) & C-SCLC (\%) & $P$ \\
\hline Total & $20,84 I(100)$ & $400(100)$ & \\
\hline Age & & & 0.158 \\
\hline$<65$ & $8599(41.3)$ & 151 (37.8) & \\
\hline$\geq 65$ & $12,242(58.7)$ & $249(62.3)$ & \\
\hline Race & & & 0.030 \\
\hline White & $17,990(86.3)$ & $328(82.0)$ & \\
\hline Black & $1975(9.5)$ & $53(13.3)$ & \\
\hline Others & $876(4.2)$ & $19(4.8)$ & \\
\hline Sex & & & 0.018 \\
\hline Female & $10,469(50.2)$ & $177(44.3)$ & \\
\hline Male & $10,372(49.8)$ & $223(55.8)$ & \\
\hline T stage & & & $<0.001$ \\
\hline T0 & $266(1.3)$ & $\mathrm{I}(0.3)$ & \\
\hline TI & $2529(12.1)$ & $86(21.5)$ & \\
\hline T2 & $545 \mathrm{I}(26.2)$ & $115(28.8)$ & \\
\hline T3 & $4542(21.8)$ & $89(22.3)$ & \\
\hline $\mathrm{T} 4$ & $8053(38.6)$ & $109(27.3)$ & \\
\hline N stage & & & $<0.001$ \\
\hline No & $3049(14.6)$ & $136(34.0)$ & \\
\hline $\mathrm{NI}$ & $1510(7.2)$ & $34(8.5)$ & \\
\hline N2 & II,6I4 (55.7) & $160(40.0)$ & \\
\hline N3 & $4668(22.4)$ & $70(17.5)$ & \\
\hline Surgery & & & $<0.001$ \\
\hline Yes & $495(2.4)$ & $106(26.5)$ & \\
\hline No & $20,346(97.6)$ & $294(73.5)$ & \\
\hline Bone metastasis & & & $<0.001$ \\
\hline Yes & $4853(23.3)$ & $62(15.5)$ & \\
\hline No & I5,988 (76.7) & $338(84.5)$ & \\
\hline Brain metastasis & & & 0.008 \\
\hline Yes & $3479(16.7)$ & 47 (II.8) & \\
\hline No & $17,362(83.3)$ & $353(88.3)$ & \\
\hline Liver metastasis & & & $<0.001$ \\
\hline Yes & $6291(30.2)$ & $64(16.0)$ & \\
\hline No & I4,550 (69.8) & $336(84.0)$ & \\
\hline Lung metastasis & & & 0.291 \\
\hline Yes & $2994(14.4)$ & $50(12.5)$ & \\
\hline No & I7,847 (85.6) & $350(87.5)$ & \\
\hline
\end{tabular}

\section{Risk Factor Analysis of Metastasis Pattern for Patients with c-SCLC Compared with SCLC}

In the present study, we mainly focused on the risk of metastasis pattern for patients with c-SCLC compared 


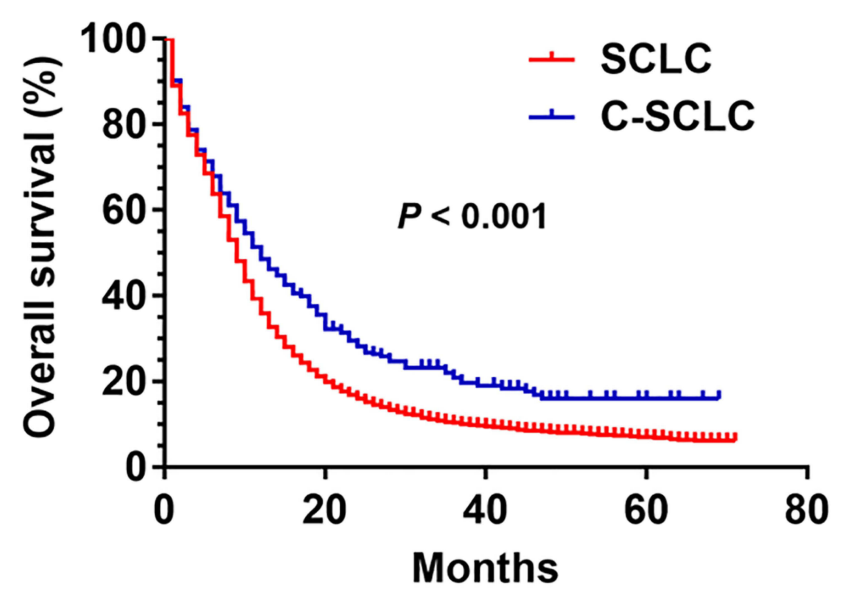

Figure I Survival curves for patients with C-SCLC and SCLC before PSM ( $<<$ $0.001)$.

with SCLC. Multivariate logistic regression analysis was used to analyze the influence variables including lymph node metastasis, bone metastasis, brain metastasis, liver metastasis and lung metastasis in c-SCLC and SCLC. Compared with SCLC patients, the risk of lymph node metastasis (OR: 0.555; 95\% CI: 0.439-0.703; P < 0.001) and liver metastasis (OR: 0.591; 95\% CI: 0.448-0.779; $\mathrm{P}<$ 0.001 ) was lower among patients with c-SCLC. However, there was no significant difference in the other risk factors including bone metastasis, brain metastasis and lung metastasis (all, $\mathrm{P}>0.05$ ). The specific details are shown in Supplementary Table 1.

\section{Discussion}

Previous studies indicated that c-SCLC accounted for 2$24 \%$ among all SCLC patients. ${ }^{5,10,11}$ SCLC patients had worse survival than NSCLC patients; however, there were few studies on prognosis of patients with c-SCLC. Various studies reported that the clinical outcomes of patients diagnosed with c-SCLC differ significantly, and the median OS ranged from 9.4 to 27 months for c-SCLC patients with I-IV stage. ${ }^{12-15}$ Baker et al showed that patients with c-SCLC had a fairly favorable prognosis. ${ }^{16}$ However, Radice et al showed that patients with SCLC had better survival than those with c-SCLC. ${ }^{6}$ Similarly, Zhang et al also indicated that the mixed NSCLC components within c-SCLC had a significant influence on the survival. ${ }^{14}$ In contrast, several researches reported that no difference in survival was found between c-SCLC and pure SCLC. ${ }^{13,17}$ The survival difference between c-SCLC and SCLC has been controversial. Therefore, in the present study, we analyzed the clinicopathological characteristic and
Table 2 Propensity Score Matching Was Conducted Among Small Cell Lung Cancer and Combined Small Cell Lung Cancer Patients

\begin{tabular}{|c|c|c|c|}
\hline Variables & SCLC (\%) & C-SCLC (\%) & $P$ \\
\hline Total & $400(100)$ & $400(100)$ & \\
\hline Age & & & 0.714 \\
\hline$<65$ & $146(36.5)$ & I5I (37.8) & \\
\hline$\geq 65$ & $254(63.5)$ & $249(62.3)$ & \\
\hline Race & & & 0.865 \\
\hline White & $332(83.0)$ & $328(82.0)$ & \\
\hline Black & $52(13.0)$ & $53(13.3)$ & \\
\hline Others & $16(4.0)$ & $19(4.8)$ & \\
\hline Sex & & & 0.943 \\
\hline Female & $176(44.0)$ & $177(44.3)$ & \\
\hline Male & $224(56.0)$ & $223(55.8)$ & \\
\hline T stage & & & 0.999 \\
\hline T0 & $\mathrm{I}(0.3)$ & $\mathrm{I}(0.3)$ & \\
\hline TI & $83(20.8)$ & $86(21.5)$ & \\
\hline $\mathrm{T} 2$ & 117 (29.3) & $115(28.8)$ & \\
\hline T3 & $88(22.0)$ & $89(22.3)$ & \\
\hline $\mathrm{T} 4$ & III (27.8) & $109(27.3)$ & \\
\hline N stage & & & 0.998 \\
\hline No & $136(34.0)$ & $136(34.0)$ & \\
\hline $\mathrm{NI}$ & $33(8.3)$ & $34(8.5)$ & \\
\hline N2 & $162(40.5)$ & $160(40.0)$ & \\
\hline N3 & $69(17.3)$ & $70(17.5)$ & \\
\hline Surgery & & & $<0.001$ \\
\hline Yes & $24(6.0)$ & $106(26.5)$ & \\
\hline No & $376(94.0)$ & $294(73.5)$ & \\
\hline Bone metastasis & & & \\
\hline Yes & $62(15.5)$ & $62(15.5)$ & 1.000 \\
\hline No & $338(84.5)$ & $338(84.5)$ & \\
\hline Brain metastasis & & & \\
\hline Yes & $43(10.8)$ & 47 (II.8) & 0.654 \\
\hline No & $357(89.3)$ & $353(88.3)$ & \\
\hline Liver metastasis & & & \\
\hline Yes & $64(16.0)$ & $64(16.0)$ & 1.000 \\
\hline No & $336(84.0)$ & $336(84.0)$ & \\
\hline Lung metastasis & & & 0.663 \\
\hline Yes & 46 (II.5) & $50(12.5)$ & \\
\hline No & $354(88.5)$ & $350(87.5)$ & \\
\hline
\end{tabular}

survival difference between c-SCLC and SCLC. Notably, we also analyzed factors, particularly on metastasis sites, that may possibly contribute to their survival difference.

In the present study, to address this issue, we have screened a total of 20,841 SCLC and 400 c-SCLC using 
Table 3 Cox Regression Analyses of Prognostic Factors for Overall Survival for Patients with Small Cell Lung Cancer and Combined Small Cell Lung Cancer

\begin{tabular}{|c|c|c|c|c|}
\hline \multirow[t]{2}{*}{ Variables } & \multicolumn{2}{|c|}{ Univariate Analysis } & \multicolumn{2}{|c|}{ Multivariate Analysis } \\
\hline & Wald $\chi^{2}$ & $P$ & HR (95\% CI) & $P$ \\
\hline $\begin{array}{l}\text { Age } \\
\qquad 65 \\
\geq 65\end{array}$ & 368.60 & $<0.0001$ & $\begin{array}{l}\text { Reference } \\
\text { I.424 (I.380-1.469) }\end{array}$ & $\begin{array}{l}<0.0001 \\
<0.0001\end{array}$ \\
\hline $\begin{array}{l}\text { Race } \\
\text { White } \\
\text { Black } \\
\text { Others }\end{array}$ & 14.53 & 0.001 & $\begin{array}{l}0.94 I(0.893-0.991) \\
0.922(0.856-0.995)\end{array}$ & $\begin{array}{l}0.011 \\
0.022 \\
0.035\end{array}$ \\
\hline $\begin{array}{l}\text { Sex } \\
\text { Female } \\
\text { Male }\end{array}$ & 110.88 & $<0.0001$ & $1.123(1.090-1.158)$ & $\begin{array}{l}<0.0001 \\
<0.0001\end{array}$ \\
\hline $\begin{array}{l}\text { Cancer type } \\
\text { SCLC } \\
\text { C-SCLC }\end{array}$ & 35.35 & $<0.0001$ & $0.950(0.842-1.073)$ & $\begin{array}{l}0.411 \\
0.411\end{array}$ \\
\hline $\begin{array}{l}\text { T stage } \\
\text { T0 } \\
\text { TI } \\
\text { T2 } \\
\text { T3 } \\
\text { T4 }\end{array}$ & 312.94 & $<0.0001$ & $\begin{array}{l}\text { Reference } \\
0.926(0.800-1.073) \\
1.169(1.014-1.348) \\
1.217(1.055-1.405) \\
1.243(1.079-1.433)\end{array}$ & $\begin{array}{l}<0.0001 \\
0.308 \\
0.032 \\
0.007 \\
0.003\end{array}$ \\
\hline $\begin{array}{l}\text { N stage } \\
\text { N0 } \\
\text { NI } \\
\text { N2 } \\
\text { N3 }\end{array}$ & 259.52 & $<0.0001$ & $\begin{array}{l}\text { Reference } \\
0.974(0.907-1.045) \\
1.169(1.116-1.225) \\
1.174(1.113-1.239)\end{array}$ & $\begin{array}{l}<0.0001 \\
0.456 \\
<0.0001 \\
<0.0001\end{array}$ \\
\hline $\begin{array}{c}\text { Surgery } \\
\text { Yes } \\
\text { No }\end{array}$ & 295.21 & $<0.0001$ & $\begin{array}{l}\text { Reference } \\
1.927 \text { (I.7|7-2.163) }\end{array}$ & $\begin{array}{l}<0.0001 \\
<0.0001\end{array}$ \\
\hline $\begin{array}{l}\text { Bone metastasis } \\
\text { No } \\
\text { Yes }\end{array}$ & 607.88 & $<0.0001$ & $\begin{array}{l}\text { Reference } \\
1.136(1.093-1.180)\end{array}$ & $\begin{array}{l}<0.0001 \\
<0.0001\end{array}$ \\
\hline $\begin{array}{l}\text { Brain metastasis } \\
\text { No } \\
\text { Yes }\end{array}$ & 396.61 & $<0.0001$ & $\begin{array}{l}\text { Reference } \\
1.415(1.360-1.472)\end{array}$ & $\begin{array}{l}<0.0001 \\
<0.0001\end{array}$ \\
\hline $\begin{array}{l}\text { Liver metastasis } \\
\text { No } \\
\text { Yes }\end{array}$ & 1829.23 & $<0.0001$ & $\begin{array}{l}\text { Reference } \\
\text { I.789 (I.727-1.854) }\end{array}$ & $\begin{array}{l}<0.0001 \\
<0.0001\end{array}$ \\
\hline $\begin{array}{l}\text { Lung metastasis } \\
\text { No } \\
\text { Yes }\end{array}$ & 459.14 & $<0.0001$ & $\begin{array}{l}\text { Reference } \\
1.232(|.| 80-\mid .286)\end{array}$ & $\begin{array}{l}<0.0001 \\
<0.0001\end{array}$ \\
\hline
\end{tabular}

the SEER database. We firstly demonstrated that patients diagnosed with c-SCLC had better survival than those diagnosed with pure SCLC. However, multivariate Cox regression analyses found that cancer type was not an independent prognostic factor in OS after adjusting for clinicopathological variables. Further, using the 
A

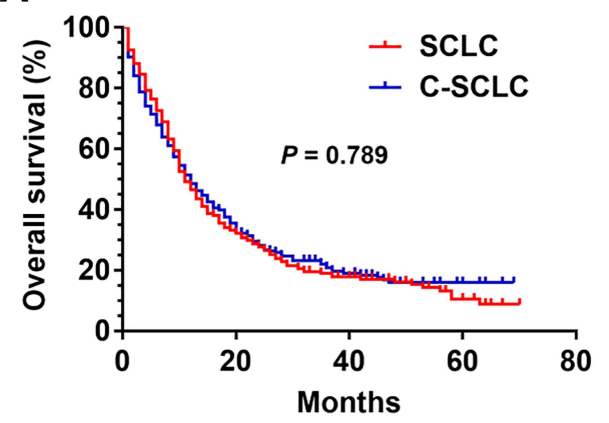

B

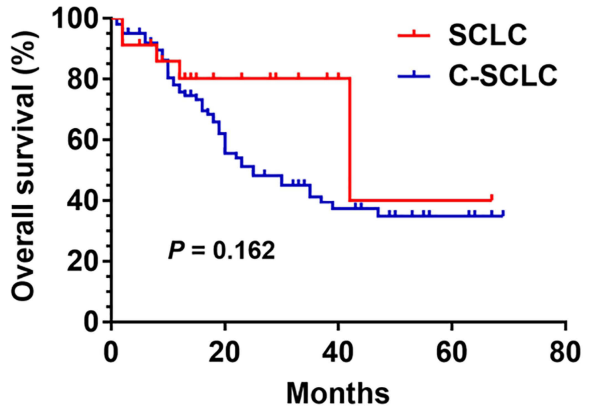

Figure 2 (A) Survival curves for patients with c-SCLC and SCLC after PSM ( $=0.789)$. (B) Survival curves for $c-S C L C$ and SCLC patients after surgery $(P=0.162)$.

multivariate Cox regression analysis, we analyzed the factors influencing the survival of $400 \mathrm{c}$-SCLC patients. We found that liver and lung metastasis were not prognostic factors for c-SCLC patients. However, patients with liver metastasis in SCLC had the worst prognosis, as reported in our previous study. ${ }^{18}$ This may be an important reason that c-SCLC patients had better survival compared with SCLC patients.

In order to better observe the influence of clinical variables on the prognosis, PSM method was used for 1:1 matching analysis. PSM-matched age, race, sex, T stage, $\mathrm{N}$ stage, bone metastasis, brain metastasis, liver metastasis and lung metastasis. And surgery was not included in the matching variable. However, our results demonstrated that there was no significant difference in survival between patients with c-SCLC and pure SCLC after PSM, suggesting that the survival benefits of c-SCLC patients may be associated with clinical variables upon diagnosis and metastasis mode, whereas they may not be associated with follow-up treatment. Since c-SCLC patients' survival had little association with treatment, we took the variable of surgery as an example and found that there was no significant difference in survival between patients undergoing surgery and those without. Therefore, we concluded that the survival may not be linked with follow-up treatment among c-SCLC patients. In the present study, we mainly focused on the odds ratio (OR) of metastasis sites in c-SCLC compared with SCLC. In multivariate logistic regression analysis, we found that the risk of lymph node and liver metastasis of c-SCLC patients was lower compared with SCLC patients, whereas there was no statistical significance in bone, brain and lung metastasis. This suggested that c-SCLC patients' survival benefit may be attributed to fewer liver and $\mathrm{N}$ metastases. This may serve as a feasible explanation for the prolonged survival of c-SCLC patients over SCLC patients. Nevertheless, it has not to be neglected that there might be other factors, such as race, sex, T stage, surgery contributing to the survival difference between SCLC and c-SCLC.

However, this study has some limitations that should be noted. First, this was a retrospective study. Secondly, some other variables, including type of surgery, other treatments affecting the prognosis and smoking history were not included in the analysis. Thus, the response to the different regimens was difficult to accurately evaluate between SCLC and c-SCLC. Thirdly, not all the SCLC and c-SCLC patients have undergone surgical resection in the present study. Since pathological confirmation by biopsies may not be as convincing as diagnosis by surgical resection, therefore some c-SCLC cases would have been mistakenly categorized as SCLC. Furthermore, since detailed information on the subtype of c-SCLC is not available from SEER database, the analysis of the subtype of c-SCLC associated with prognosis could not be conducted. These aspects would be improved in our future studies. Additionally, due to the small samples of c-SCLC, these results may be biased. Therefore, further large-scale prospective clinical studies are required to confirm these recommendations.

\section{Conclusions}

We showed that c-SCLC differed from SCLC in many clinicopathological features. We also demonstrated the prognosis of c-SCLC did not significantly differ from that of SCLC if clinicopathological characteristics are controlled. Better prognosis for c-SCLC patients over SCLC patients may be ascribed to fewer liver and lymph node metastases upon diagnosis. From a clinical standpoint, we should take liver and lymph node metastases into account in the comparison of prognosis of c-SCLC and SCLC patients. The status of liver and lymph node metastases should be monitored in the follow-up of SCLC and c-SCLC patients to better 
evaluate prognosis. Due to distinct clinicopathological features and risk factors, future clinical trials for SCLC and c-SCLC may be conducted differentially.

\section{Data Sharing Statement}

The datasets used and/or analyzed during the present study are available from the corresponding authors on reasonable requests.

\section{Ethics Approval and Consent to Participate}

Our study was mainly based on the SEER database. Institutional approval and patient consents were not needed due to open access to the public database, according to the stipulations by Shandong Cancer Hospital and Institute, Fudan University Shanghai Cancer Center.

\section{Acknowledgments}

We would like to express our appreciation to European Society for Medical Oncology and World Conference on Lung Cancer for presenting the abstract of some of our results from the study.

\section{Author Contributions}

All authors made a significant contribution to the work reported, whether that is in the conception, study design, execution, acquisition of data, analysis and interpretation, or in all these areas; took part in drafting, revising or critically reviewing the article; gave final approval of the version to be published; have agreed on the journal to which the article has been submitted; and agree to be accountable for all aspects of the work. Chenyue Zhang and Xiaoling Shang are the co-first authors for this study; Chenyue Zhang and Haiyong Wang are the corresponding authors for this study.

\section{Disclosure}

The authors declare no conflicts of interest in this work.

\section{References}

1. Govindan R, Page N, Morgensztern D, et al. Changing epidemiology of small-cell lung cancer in the United States over the last 30 years: analysis of the surveillance, epidemiologic, and end results database. J Clin Oncol. 2006;24:4539-4544.

2. Owonikoko TK, Ragin CC, Belani CP, et al. Lung cancer in elderly patients: an analysis of the surveillance, epidemiology, and end results database. J Clin Oncol. 2007;25(35):5570-5577. doi:10.120 0/JCO.2007.12.5435
3. Eskandar A, Ahmed A, Daughtey M, et al. Racial and sex differences in presentation and outcomes of small cell lung cancer in the United States: 1973 to 2010. Chest. 2015;147(4):e164-e165. doi:10.1378/ chest.14-3051

4. Travis WD, Coby TV, Corrin B, et al. World Health Organization International Histological Classification of Tumors. 3rd ed. Berlin: Springer; 1999.

5. Babakoohi S, Fu P, Yang M, et al. Combined SCLC clinical and pathologic characteristics. Clin Lung Cancer. 2013;14(2):113-119. doi:10.1016/j.cllc.2012.07.002

6. Radice PA, Matthews MJ, Ihde DC, et al. The clinical behavior of "mixed" small cell/large cell bronchogenic carcinoma compared to "pure" small cell subtypes. Cancer. 1982;50:2894-2902. doi:10.1002/1097-0142(19821215)50:12<2894::AID-CNCR2820501232>3.0.CO;2-G

7. Takenaka $T$, Takenoyama M, Inamasu $E$, et al. Role of surgical resection for patients with limited disease-small cell lung cancer. Lung Cancer. 2015;88(1):52-56. doi:10.1016/j.lungcan.2015.01.010

8. Takei H, Kondo H, Miyaoka E, et al. Surgery for small cell lung cancer: a retrospective analysis of 243 patients from Japanese lung cancer registry in 2004. $J$ Thorac Oncol. 2014;9(8):1140-1145. doi:10.1097/JTO.00000000000000226

9. Surveillance, Epidemiology, and End Results (SEER) program. SEER*stat database: incidence-SEER 18 regs research data + hurricane Katrina impacted Louisiana cases, Nov 2018 sub (1975-2016 varying) linked to county attributes - total U.S., 1969-2017 counties. National Cancer Institute, DCCPS, Surveillance Research Program, released April 2019. Based on the November 2018 submission. Available from: www.seer.cancer.gov. Accessed October 6, 2021.

10. Mangum MD, Greco FA, Hainsworth JD, et al. Combined small-cell and non-small-cell lung cancer. J Clin Oncol. 1989;7(5):607-612. doi:10.1200/JCO.1989.7.5.607

11. Hirsch FR, Osterlind K, Hansen HH. The prognostic significance of histopathologic subtyping of small cell carcinoma of the lung according to the classification of the World Health Organization. A study of 375 consecutive patients. Cancer. 1983;52(11):2144-2150. doi:10.1002/1097-0142(19831201)52:11<2144::AIDCNCR2820521128 $>3.0 . \mathrm{CO} ; 2-\mathrm{N}$

12. Moniodis A, Racila E, Divo M. Case report: combined small cell lung cancer in a lung transplant recipient. Transplant Proc. 2015;47 (3):852-854. doi:10.1016/j.transproceed.2015.01.019

13. Wallace AS, Arya M, Frazier SR, et al. Combined small-cell lung carcinoma: an institutional experience. Thorac Cancer. 2014;5 (1):57-62. doi:10.1111/1759-7714.12059

14. Zhang C, Yang H, Zhao H, et al. Clinical outcomes of surgically resected combined small cell lung cancer: a two institutional experience. J Thorac Dis. 2017;9:151-158. doi:10.21037/jtd.201 7.01.07

15. Kim YH, Mishima M, Date H. "Combined" small cell and "pure" small cell lung cancer: is there a clinical difference? Med Oncol. 2013;30(2):600. doi:10.1007/s12032-013-0600-1

16. Baker RR, Ettinger DS, Ruckdeschel JD, et al. The role of surgery in the management of selected patients with small-cell carcinoma of the lung. J Clin Oncol. 1987;5(5):697-702. doi:10.1200/JCO.1987.5.5.697

17. Nicholson SA, Beasley MB, Brambilla E, et al. Small cell lung carcinoma (SCLC): a clinicopathologic study of 100 cases with surgical specimens. Am J Surg Pathol. 2002;26(9):1184-1197. doi:10.1097/00000478-200209000-00009

18. Shang X, Lin J, Li Z, et al. Radiotherapy may improve survival of ES-SCLC with distant metastasis only for patients with one metastatic site: a population-based study. Oncol Lett. 2020;19(1):139-146. 


\section{Publish your work in this journal}

The International Journal of General Medicine is an international, peer-reviewed open-access journal that focuses on general and internal medicine, pathogenesis, epidemiology, diagnosis, monitoring and treatment protocols. The journal is characterized by the rapid reporting of reviews, original research and clinical studies across all disease areas. The manuscript management system is completely online and includes a very quick and fair peer-review system, which is all easy to use. Visit http://www.dovepress.com/ testimonials.php to read real quotes from published authors.

Submit your manuscript here: https://www.dovepress.com/international-journal-of-general-medicine-journal 